\title{
Ante-grade ureteric stenting, retrospective experience in managing 89 patients: Indications, complications and outcome
}

\author{
Anthony Kodzo-Grey Venyo ${ }^{1}$, Tessa Hanley ${ }^{2}$, Michael Barrett ${ }^{1}$, Ali Nawaz Khan ${ }^{3}$ \\ 1. Department of Urology, North Manchester General Hospital, Manchester, United Kingdom. 2. Department of \\ Anaesthesia, North Manchester General Hospital, Manchester, United Kingdom. 3. Department of Radiology, North \\ Manchester General Hospital, Manchester, United Kingdom.
}

Correspondence: Anthony Kodzo-Grey Venyo. Address: Department of Urology, North Manchester General Hospital, Delaunays Road, Manchester, United Kingdom. E-mail: akodzogrey@yahoo.co.uk

Received: May 5, 2014

DOI : $10.5430 / j b g c . v 4 n 3 p 47$
Online Published: July 28, 2014

URL: http://dx.doi.org/10.5430/jbgc.v4n3p47

\section{Abstract}

Background: Over the past three decades there has been a remarkable increase in interventional uro-radiological procedures in the developed countries. Long-term drainage of the obstructed upper urinary tract may be achieved by percutaneous nephrostomy or ureteric stenting. Previously most ureteric stents were inserted by the retrograde approach at cystoscopy and these procedures have required the use of general or spinal anesthesia. With the development of a nephrostomy service in most hospitals throughout the world ante-grade ureteric stenting procedures are being carried out by trained interventional radiologists in the radiology department without the need for a general or spinal anesthesia.

Aims/materials and methods: To review the records of all 89 patients who had insertion of ante-grade ureteric stents for ureteric obstruction between February 2010 and January 2013 in order to document the following: The causes of obstruction; The side (left, right or both sides) and site of the ureteric obstruction (upper, mid or lower ureter); The sex and ages of the patients; Success or failure of the procedure; Complications of procedure; The management of the complications.

Results: Ante-grade ureteric stenting was successful in 105 out of 121 procedures (86.7\%) which 89 patients underwent. Out of 105 successful ante-grade stenting procedures carried out, 103 were successful at first attempt, another two were successful at second attempt and one successful at a third attempt, making it a total of 105 successful procedures. There were minimal complications.

Conclusion and recommendation: Ante-grade ureteric stenting is a safe procedure with minimal complications. Every radiology unit should have a trained interventional radiologist capable of performing ante-grade ureteric stenting.

\section{Key words}

Nephrostomy, Ante-grade ureteric stenting, Complications, Upper urinary tract obstruction, Retro-grade ureteric stenting, Interventional radiologist

\section{I ntroduction}

Patients who require temporary or long term urinary drainage for obstruction of the upper urinary tract may be managed by a number of procedures some of which include percutaneous nephrostomy insertion, ante-grade ureteric stent 
insertion, retrograde ureteric stent insertion, ileal conduit construction, ureterostomy procedure as well as uretero-ureteric anastomosis ${ }^{[1]}$. The first two aforementioned procedures can be performed with the use of local anesthesia, sedation and analgesia in the radiology department but the remaining procedures do require the use of general anesthesia in the operating theatre and require the patients to be fit to undergo general anesthesia ${ }^{[1]}$. The management of ureteric obstruction depends upon the underlying pathology, type/cause of obstruction/stricture and also the patient's preference and whether or not the patient is fit to under-go anesthesia ${ }^{[1]}$. Percutaneous nephrostomy (PCN) can provide temporary or permanent drainage of an obstructed urinary system ${ }^{[1]}$. Patients with benign/malignant ureteric strictures are often treated with ureteric stenting via an ante-grade, retrograde or combined approach ${ }^{[1]}$. Sometimes retrograde ureteric stenting may be abandoned in theatre due to the inability of the surgeon to advance the guide-wire by the retrograde approach beyond the point of obstruction; in such cases the only options left are insertion of a percutaneous nephrostomy plus or minus an attempt at insertion of the ureteric stent by means of the ante-grade approach ${ }^{[1]}$. Long-term urinary drainage in patients with ureteric obstruction due to benign or malignant strictures or due to other extra-ureteric causes of ureteric obstruction is often treated with ureteric stenting at the same time as insertion of a nephrostomy or at times as a follow-up procedure by inserting the ureteric stent by the ante-grade approach through the nephrostomy. The insertion of a ureteric stent renders the patient tubeless and avoids the use of a nephrostomy bag which has to be changed regularly and also avoids nephrostomy tube associated problems thus provides better quality of life ${ }^{[1]}$. It is also possible for a ureteric stent to be inserted in a patient who is temporarily not fit to undergo general anesthesia ${ }^{[1]}$. Venyo and Bakir reported a success rate of 92.5\% in their initial 30 patients who underwent ante-grade ureteric stent insertions. This paper reviews the subsequent experience in the use of ante-grade ureteric stenting through a percutaneous nephrostomy in a larger number of patients who underwent similar procedures between February 2010 and January 2013.

\section{Patients, materials and methods}

\subsection{Patients}

The clinical records, laboratory results and radiological records from the PACS system of all the eighty-nine patients (52 male; 37 female) aged $<30$ to $>90$ years (mean age 66.2 years) who underwent a total of 121 insertion of ante-grade ureteric stenting for obstruction of the upper urinary tract were reviewed (see Figure 1 for the causes of obstruction requiring ante-grade ureteric stenting). The following data were recorded for each patient: the indication for the procedure/ cause of obstruction, the side and site of obstruction, the sex and age of the patient, the outcome (success/failure) of the procedure, complication(s) of the procedure and the management of the complication.

Figure 1. Causes of upper ureteric obstruction

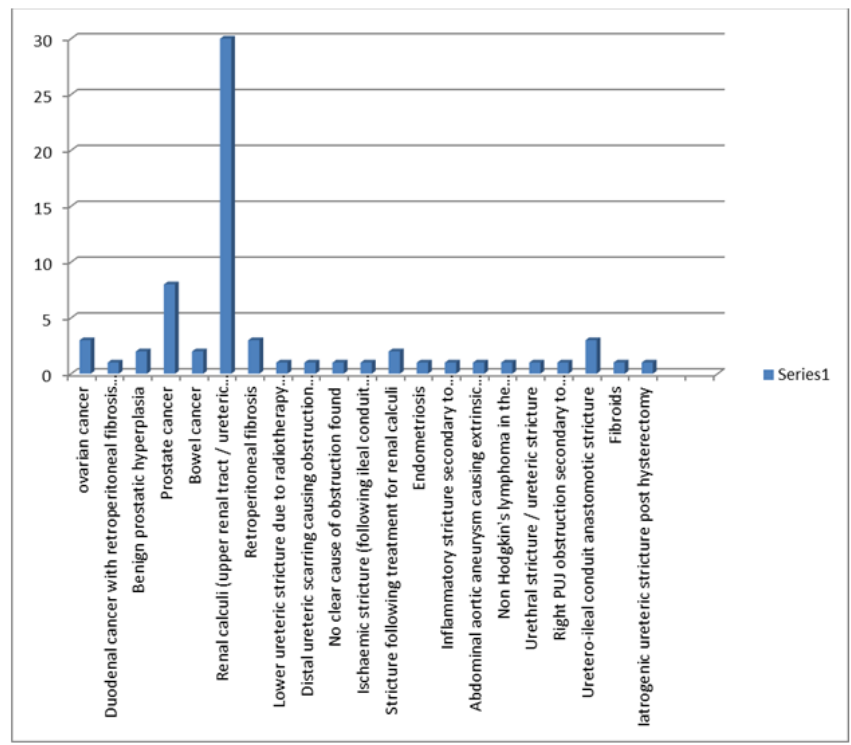


Age: Range 18 to 91 (Mean 66.2) years (see Figure 2);

Sex: Male 56 patients; Female 38 patients; Total 89 patients.

Figure 2. Age distribution of patients who underwent insertion of ante-grade ureteric stent

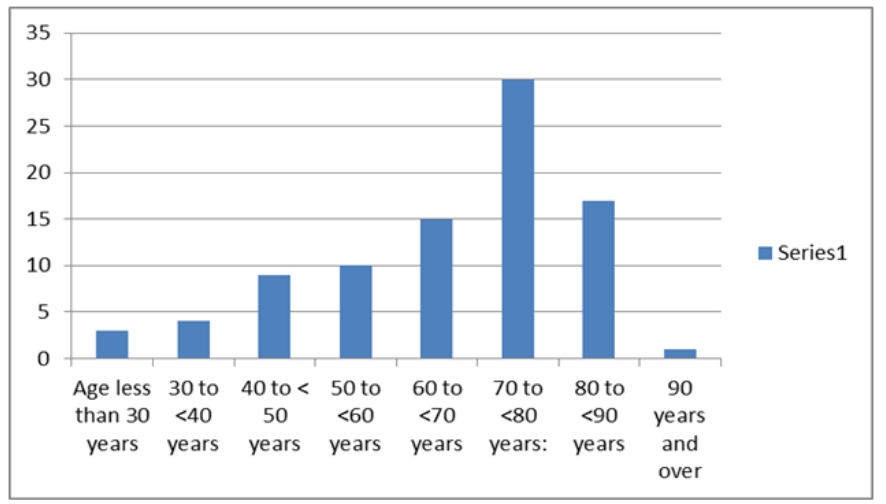

\subsection{Materials and methods}

\subsubsection{Per-cutaneous nephrostomy}

\section{Techniques}

Two different ultrasound-guided techniques: the Seldinger technique and the "one-stab technique" can be used for the insertion of percutaneous nephrostomy.

The patients are placed in prone or prone-oblique position for the procedure. It is routine to administer to the patients antibiotics prophylactically and with suspected pyonephrosis and renal stone disease. Analgesia is routinely given (for example Pethidine intramuscularly or by Intravenous titration method) as well as an anti-emetic. Coagulation screen is also routinely done and the procedure is carried out if the INR is 1.2 or below.

The selection criteria for each technique, is dependent upon the configuration of the renal collecting system. The "Seldinger technique" is usually the method of choice for non-dilated collecting systems and cases with suspected pyonephrosis. This technique is performed with fluoroscopic guidance. The "one stab" (Bonanno) technique is used without fluoroscopic guidance for moderate-to-severe dilated collecting systems.

The "Seldinger" technique involves ultrasound-guided puncture of the dilated renal collecting system with a 19-G sheathed needle, the insertion of a 0.0038 ' heavy-duty J guide-wire and serial dilatation of the tract with 6 to $10 \mathrm{~F}$ dilators up in order to accommodate 8 to $12 \mathrm{~F}$ nephrostomy catheters with or without fluoroscopic guidance (see Figures 3a-3c). With fluoroscopic guidance Urografin contrast (about $5 \mathrm{mls}$ ) is routinely administered to confirm the position of the ' $19 \mathrm{G}$ ' sheathed needle prior to the insertion of the guide-wire. Quite often either Locking-Loop Pigtail (LLP) catheters (Cook Inc, USA) or All Purpose Drainage (APD) catheters (Boston Scientific, USA) are used for nephrostomies.

The second technique which is called the "one stab technique" or the "Bonanno technique", this involves the use of ultrasound-guided "one-stab” technique using a 6 F Bonanno catheter (Beckton Dickinson UK Ltd). This 6 F pigtail Teflon catheter is mounted on a hollow $18 \mathrm{G}$ needle which has a sharp bevelled edge. Under ultrasound guidance the needle tip is inserted into the guided pelvi-calyceal system, and on obtaining urine backflow the catheter is slid over the needle into the collecting system.

The nephrostomy catheter is secured to the skin by a catheter fixation disc covered with adhesive dressings and then connected to a closed urinary drainage bag system. 


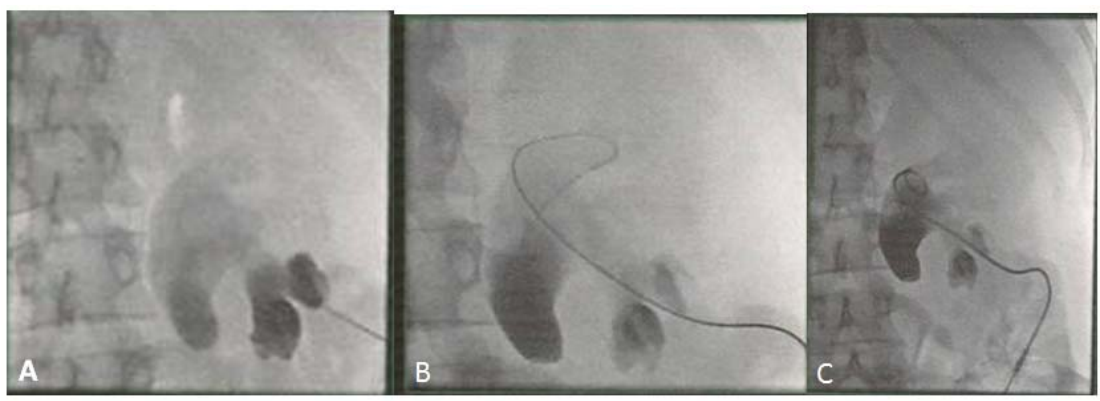

Figure 3. Some stages of insertion of nephrostomy. A: Right lower calyx was punctured with a Chiba needle; B: Amplatz guide-wire is advanced in to the right renal pelvis; C: Nephrostomy tube size 6 Fr is in situ.

\subsubsection{Ureteric stenting}

\section{Technique - ante-grade approach}

Benign/Malignant strictures or extra-luminal ureteric obstructions may be negotiated by means of a combination of wires and catheters that are passed through a nephrostomy tract under fluoroscopic control to traverse the ureteric narrowing. Usually an angled tip hydrophilic tip Terumo wire is used to negotiate through the ureteric obstructing point and it is then gradually passed into the urinary bladder. Successful entry into the bladder is confirmed fluoroscopically. In most cases a $6 \mathrm{~F}$ torque-controlled manipulation catheter is used to cannulate the stricture or constricting area and bladder (see Figures 4a-4h), nevertheless, in difficult cases a $4 \mathrm{~F}$ or $5 \mathrm{~F}$ "hydrophilic-coated Cobra catheter" is used. The Terumo wire is then removed and this is replaced by a stiffer wire (e.g. Amplatz super-stiff wire; Boston Scientific, USA) over which the ureteric stent is inserted. Sometimes when a benign stricture is encountered it can be treated by a balloon catheter dilatation or a PTFE-coated Van Andel dilator. Quite often in our unit a 6 to 8 F (Meditech, USA, Boston Scientific, USA) or 7 F (Optimed Technologies Inc. Germany) ureteric stent is used. Pursuant to the ante-grade ureteric stenting procedures, these stents are subsequently electively changed by the urologist using the retrograde approach every three to six months to avoid stent encrustation.
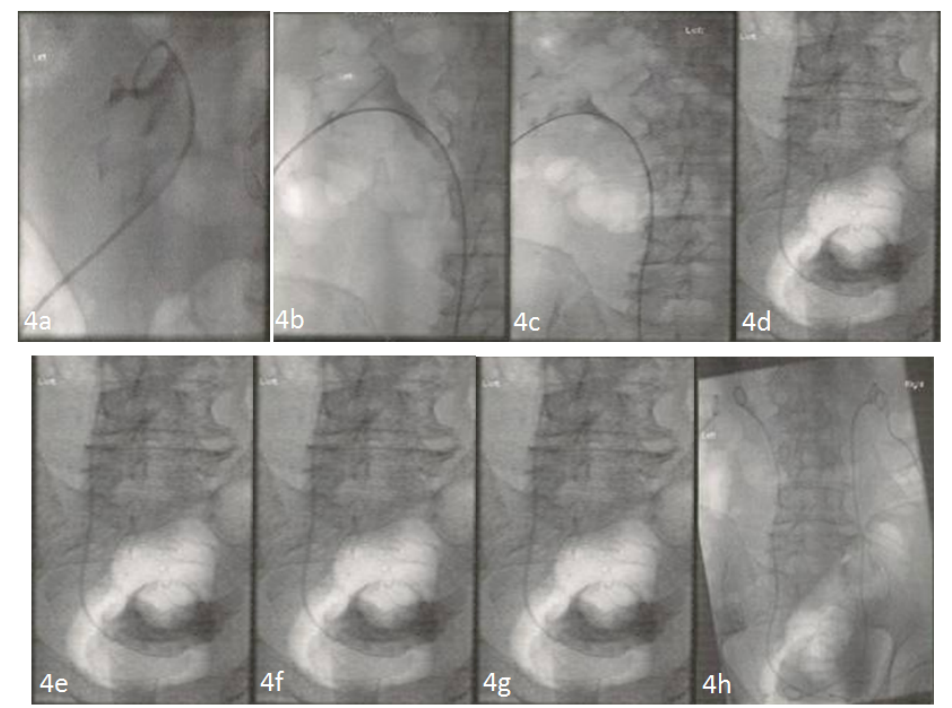

Figure 4. Some stages of insertion of ante-grade ureteric stent. 4a: Left Nephrostomy tube is in situ; 4b: Terumo Guide-wire has been inserted into left lower pole calyx, renal pelvis and left ureter (guide wire can be seen in the upper ureter; the lower ureter is not shown in this picture); 4c: Terumo guide-wire has been inserted in to the left ureter and down into to the urinary bladder (another view of 4b); 4d: Left Ante-grade ureteric stent has been inserted in to the urinary bladder (lower two thirds of stent is in the picture; 4e: This shows a left Ante-grade ureteric stent inserted into the urinary bladder over a stiff guide-wire; 4f: This shows a guide-wire which has been inserted into right lower pole calyx and in to upper right ureter; 4g: This shows a right ante-grade ureteric stent which has been inserted over a stiff guide- wire; 4h: This shows bilateral ante-grade ureteric stents, in situ. 


\section{Analysis}

The indication(s) for the procedures were recorded as well as the total number(s) involved. The following details were also worked out and recorded: Total number of male and female patients as well as the total number of procedures undertaken; Total number of procedures on each side; Total number of successful procedures and failures including reason(s) for the failures; Total number of complications and treatment of the complication(s).

\section{Results}

In total, 89 patients underwent ante-grade ureteric stent insertion. In these 89 patients, a total of 121 ante-grade stent insertions were performed.

Of the 121 stenting procedures carried out the 89 patients involved, there were 15 patients in whom there was failure of stent insertion at the first attempt. In these 15 patients, there were 16 failed attempts at ante-grade insertion. In two patients, in whom the initial attempt was unsuccessful, a subsequent second attempt was successful. In one patient, the first two attempts at ante-grade stent insertion were unsuccessful, with a successful third attempt; however, the stent had to be removed two days later because it became coiled in the pelvi-ureteric junction (PUJ).

Of the 105 successful procedures, 103 stenting procedures were successful at first attempt, two at second attempt and one at the third attempt.

The results are summarized as follows: One patient had a bladder tumor causing obstructions at the vesico-ureteric junctions (VUJs) bilaterally. The right stent was inserted at the first attempt without difficulty. With regards to the left side, the catheter and guide-wire were advanced into the bladder without problem. Then the eight French ureteric stent was advanced over the guide-wire into the bladder, which resulted in extrusion of the distal end of the catheter through the urethra. The reason for this was conjectured to have been a result of a shrunken urinary bladder. The left stent insertion was abandoned and a nephrostomy catheter inserted instead. One patient with a bladder cancer underwent bilateral stenting. Insertion of the left stent failed at the first attempt due to the grossly distended renal pelvis and acute PUJ obstruction. However a second attempt four days later was successful. One patient, who underwent bilateral stent insertion for bladder cancer, had a successful right sided stent insertion. However, the lower ureteric stricture on the left hand side was too tight and it was not possible to pass the guide-wire through the stricture. A left sided nephrostomy was left in place. One patient had a bilateral distal ureteric obstruction due to bowel cancer. During the insertion of bilateral stents the right stent was placed without complications. The left stent however was not inserted, as the guide-wire could not be passed through the tight stricture. One patient had renal calculi causing bilateral PUJ obstructions. Bilateral stenting was attempted however the right side was unsuccessful as the PUJ obstruction on this side was irregular. The position of the right nephrostomy was at the lower pole which made the angle to access the PUJ impossible. One patient with a bladder tumor had a failed left stent insertion as there was significant scarring at the left PUJ which prevented insertion of the stent. The catheter of the stent was left in situ so that the urology team might pass a guide-wire through the catheter directly. However the team decided to carry out retrograde stenting instead. One patient with bladder tumor had an attempt at left sided stenting abandoned as the 12th rib was inhibiting access and the stricture at the left VUJ was too tight. A left sided nephrostomy was left in place. One patient with metastatic prostate carcinoma had a left sided stent inserted. However, the stent fractured once the thread was removed and the upper J loop became stuck in the calyx Attempt to retrieve the stent was unsuccessful. A left sided nephrostomy was left in situ to drain the system. One patient with PUJ obstruction for which the cause was unclear had a failed left ante-grade stent insertion. The guide-wire was easily introduced; however, it later slipped out. The procedure was reattempted, but the patient was unable to tolerate the pain. Subsequent ultrasound scans failed to show any evidence of hydronephrosis and it was deemed that nephrostomy was not necessary. In one patient with a right ureteric stone causing a PUJ obstruction the tail of the stent became tangled in the nephrostomy tube during its removal. The clinician advised abandoning the procedure. An attempt was made to place a further nephrostomy, however the system was decompressed at this point and attempts were unsuccessful. In one patient with a right staghorn calculus the 
procedure was abandoned. Initially the dye injected into the catheter following PCNL did not opacify the collecting system and appeared to be outside the pelvis. Insertion of the stent via ultrasound guidance was then attempted but the patient could not tolerate the pain and the procedure was abandoned. In one patient with a right ureteric stone, the insertion of an ante-grade stent failed and was abandoned as the right PUJ was completely obstructed. The patient later underwent a nephrectomy of their non-functioning right kidney. In one patient with an ileal conduit due for intractable incontinence, the insertion of a right ante-grade stent failed because of an extended stricture along the right ureter. Despite several attempts to place a guide-wire through the previously placed drain, it was impossible to negotiate the long ischemic stricture. A right nephrostomy was inserted instead. One patient had a right hydro-ureteronephrosis following iatrogenic damage to her right ureter following a hysterectomy and bilateral salpingo-oophorectomy for fibroids. Two attempts to insert a right ante-grade stent were unsuccessful due to it being impossible to cross the distal ureteric stricture. On the third attempt the stent was successfully inserted but had to be removed after two days as it became coiled at the pelvi-ureteric junction. In one patient with a right sided renal calculus causing right vesico-ureteric junction obstruction the insertion of the right ante-grade stent failed due to being unable to cross the distal ureteric obstruction. A further attempt at ante-grade stenting was made two days later, which was successful.

Some of the causes of the obstructions, including the patient numbers affected are noted as follows (see Figure 1):

Bladder cancer: 19; Cervical cancer: 1; Ovarian cancer: 3; Cervical cancer: 1; Duodenal cancer with retroperitoneal fibrosis due to lymph node involvement: 1; Benign Prostatic Hyperplasia: 2; Prostate cancer: 8; Bowel cancer: 2; Renal calculi: 30; Retroperitoneal fibrosis: 3; Lower ureteric stricture secondary to radiotherapy treatment for prostate cancer: 1 ; Distal ureteric scarring causing obstruction from previous TURBT: 1; No clear cause of obstruction found: 1; Ischemic stricture (following ileal conduit formation): 1; Stricture following treatment for renal calculi: 2; Endometriosis: 1; Inflammatory stricture secondary to diverticulitis: 1; Abdominal aortic aneurysm causing extrinsic compression: 1; Non-Hodgkin's lymphoma in the retroperitoneal and left renal area: 1; Urethral stricture: 1; Right PUJ obstruction secondary to laparoscopic pyeloplasty: 1; Uretero-ileal conduit anastomosis stricture: 2; Stricture at uretero-ileal conduit anastomosis: 1; Fibroids: 1; Iatrogenic ureteric stricture post hysterectomy (see Figure 1).

\subsection{Age distribution}

The ages of the patients ranged from minimum age of 18 years to a maximum age of 91 years (see Figure 2).

The age distribution of the patients is outlined as follows: Age less than 30 years: $3 ; 30$ to $<40$ years: $4 ; 40$ to $<50$ years: 9; 50 to $<60$ years: 10; 60 to $<70$ years: 15 ; 70 to $<80$ years: 30; 80 to $<90$ years: 17; 90 years and over: 1 .

Age did not affect the outcome of the ante-grade stenting procedures. Patients in the various age groups tolerated the stenting procedures equally well and there was no evidence to suggest particular complications were related to any age group.

\subsection{Sex distribution}

52 male patients and 37 female patients underwent ante-grade stenting. There was no pattern of complications pertaining to either the male or female patients. All the patients tolerated the procedure well.

\subsection{Management of complications}

Sixteen out of 89 patients (18\%), who underwent insertion of 121 ante-grade ureteric stent developed complications pursuant to their ante-grade stent insertions which are summarized as follows: Three patients (3.4\% of patients) developed mild post stent insertion pain; however these were all controlled with analgesia. One patient with right PUJ obstruction secondary to right sided laparoscopic pyeloplasty, had to have the stent removed after six days due to severe stent related pain. Two patients suffered from significant storage lower urinary tract symptoms and were prescribed anticholinergics, which helped control the symptoms. Two patients reported recurrent urinary tract infections following stent insertion. One 
patient underwent left sided ante-grade stenting for distal ureteric obstruction due to bladder TCC. Subsequent nephrostogram showed contrast entering the urinary bladder, but it was felt that there was not completely free drainage past the obstructed ureter and it was changed for a retrograde stent three days after insertion. Four patients (4.5\% of patients) developed an infection within the first month of insertion and required treatment with Intravenous (IV) antibiotics. In one of these patients, US showed an enlarged swollen kidney consistent with nephritis. In a further patient with infection, the associated pain was so severe the stent had to be removed after two days. In all cases the infections were successfully treated. A further two patients had minor infections requiring oral antibiotics. No admission was necessary. One of these patients also suffered from significant stent related pain, with reflux of urine through the nephrostomy during micturition. In one patient who underwent a right ante-grade stent insertion for hydro-uretero-nephrosis following iatrogenic damage to her ureter following a total abdominal hysterectomy, two initial attempts to insert an ante-grade stent were unsuccessful. The third attempt was successful, however the stent became coiled at the PUJ and had to be removed two days after insertion.

\section{Discussion}

Per-cutaneous nephrostomy insertion was first described in 1955 by Goodwin and associates ${ }^{[3]}$ and it is an important technique for the provision of temporary or permanent/long-term drainage of an obstructed upper urinary tract or for establishing diversion of urine flow ${ }^{[3]}$.

Reported associated complications of ante-grade ureteric stenting complications include complications that are associated with nephrostomy insertion as well as the complications emanating from manipulation of guide-wire and the stent in the ureter (the ante-grade stenting procedure). On the whole, percutaneous nephrostomy insertion is a relatively safe procedure when it is performed by well-trained and very-skilled interventional radiologists. Nevertheless, a number of complications associated with nephrostomy insertion have been reported by some authors ${ }^{[4,5]}$ as follows: (1) Major complications were reported in $4 \%$ to $8 \%$ of cases and these included significant bleeding requiring blood transfusion, septicemia; and inadvertent puncturing of pleura or viscera for example the liver, colon, and spleen; (2) minor complications were reported in $3 \%$ to $15 \%$ of cases and these include retroperitoneal extravasation of urine and significant visible hematuria causing clot colic and/or catheter blockage requiring further investigations.

The commonest early complication linked with ante-grade ureteric stent insertion is septicaemia which occurred in $60 \%$ of patients. Paz et al. ${ }^{[6]}$ reported 100 patients who underwent double J ureteric insertions by the retrograde approach. They stated that all 13 patients, who had fever at the initial evaluation, underwent urgent stent insertion. Of the 87 afebrile patients (53 elective and 34 urgent insertions), 22 (25\% developed fever ( $>$ or $=38$ degrees $\mathrm{C}$ ) after the procedure. Fever developed in 19 (56\%) of the 34 urgent insertion group who were not originally febrile as opposed to 3 (6\%) of the elective insertion group $(P<0.001)$. Six of these 22 patients $(27 \%)$ had a positive urine culture after insertion. None had a positive blood culture, including 13 patients who had the insertion whilst febrile.

Rao et al. ${ }^{[7]}$ performed a retrospective audit of 165 ante-grade double $\mathrm{J}(\mathrm{JJ})$ ureteric stent insertions. They reported that all in all, five (3\%) patients, who had delayed insertion of ante-grade ureteric stent following nephrostomy insertion, the procedure was complicated by silent ureteric perforation and an extra-anatomic placement of ureteric stent. Rao et al. ${ }^{\text {[7] }}$ also stated that the aforementioned complications had delayed manifestations, which included two retroperitoneal abscesses, a pelvic urinoma, a case each of uretero-rectal fistula, and uretero-vaginal fistula. Furthermore, Rao et al. ${ }^{[7]}$ iterated that the risk factors for ureteric perforation include previous pelvic malignancy, pelvic surgery, pelvic radiation, and a history of ureteric manipulation. Rao et al. ${ }^{[7]}$ concluded that: Ante-grade ureteric double J stenting is a procedure which is not without complications.

Liatsikos et al. ${ }^{[8]}$ reported their long-term experience with palliative-treatment for extrinsic malignant obstruction with per-cutaneous placement of metal mesh stents. A total of 119 ureters were managed. With regard to the results, Liatsikos ${ }^{[8]}$ reported that: The technical success rate of percutaneous ante-grade insertion of ureteral self-expandable metal mesh stents 
was $100 \%$. Renal biochemistry normalized and hydronephrosis gradually resolved one to two weeks following the stent insertion. The median follow-up was 15 months (range 8 to 38 months). Hyperplastic reaction and/or encrustation, or tumor ingrowth developed in 45 stents. Secondary intervention, such as repeat balloon dilation and coaxial stenting was performed to improve patency. Migration was found in 13 metal stents. The primary and secondary patency rates during follow-up were $51.2 \%$ and $62.1 \%$, respectively. A double pigtail or external-internal stent was inserted in 45 cases in which secondary interventions did not ensure patency. Liatsikos et al. ${ }^{[8]}$ concluded that: Internal drainage of extrinsic malignant ureteral obstruction with metal stents provides long-term decompression of the upper urinary tract in select cases.

Kim and Park ${ }^{[9]}$ evaluated seven patients who underwent ante-grade ureteric stent insertion with the use of pull-through technique due to tight ureteric stricture. Kim and Park ${ }^{[9]}$ reported that: Double-J ureteric stent was successfully placed in all seven patients by means of the pull-through technique. Complications included abdominal/flank pain $(\mathrm{n}=7)$, visible hematuria $(n=5)$, and elevated blood pressure $(n=1)$. All the complications were relieved within three days after the procedure and conservative management.

Watson and Patel ${ }^{[10]}$ evaluated the success rate and cost efficiency of primary ante-grade ureteric stenting (ante-grade ureteric stent insertion as a single procedure without preliminary drainage) in 38 patients (50 ureters) with obstructive hydronephrosis, of acute or chronic onset and of benign / malignant origin. They compared patients who were successfully primarily stented (group 1) with a group of patients who were stented as a traditional two-stage procedure (group 2). With regard to the results, Watson and Patel ${ }^{[6]}$ reported that: Forty out of fifty (80\%) ureters were considered primary stent successes. There was a minimum saving of pound 800 per successful primary stent insertion. The cost-effectiveness of a primary ante-grade stenting strategy was 1,229 pounds versus 2,093 pounds for secondary stenting. Watson and Patel ${ }^{[10]}$ concluded that: In carefully selected patients, the majority of obstructed ureters can be primarily stented using simple equipment. The reduced hospital stay and over-all success rate significantly improves the cost competitiveness of antegrade ureteric stent insertion.

Hausegger and Portugaller ${ }^{[11]}$ reported their experience with regard to insertion of percutaneous nephrostomy and placement of ante-grade ureteric stenting as follows: Percutaneous nephrostomy insertions were undertaken with ultrasound-scan/fluoroscopic guidance with a success rate of more than $90 \%$.The complication rate was about $10 \%$ for major and minor complications together and only 4 to $5 \%$ for major complications. Hausegger and Portugaller ${ }^{[11]}$ iterated that in the case of severe infection and bleeding pursuant to nephrostomy insertion, double $\mathrm{J}$ (JJ) stent insertion may be contraindicated as long as there is no sufficient concomitant drainage via the percutaneous nephrostomy. Hausegger and Portugaller ${ }^{[11]}$ recommended that lower urinary tract dysfunction should be excluded before stent placement.

Borelli Palanca et al. ${ }^{[12]}$ reported twenty-four patients with urinary obstruction, in which twenty-seven ante-grade ureteric stent (double J) insertions were attempted (in six patients the obstruction was bilateral and in three patients they failed). Borelli Palanca et al. ${ }^{[12]}$ stated that: They achieved a 90\% success rate. A case of peri-renal hematoma occurred after insertion of nephrostomy and this was the only relevant complication they encountered. Borelli Palanca et al. ${ }^{[12]}$ concluded that ante-grade ureteric stent insertion is a good alternative which, under several circumstances, the conventional retrograde insertion of ureteric stent fails.

Sharma et al. ${ }^{[13]}$ reported 41 patients, who underwent insertion of ante-grade ureteric stents. Sharma et al. ${ }^{[13]}$ reported that: The over-all success rate for ante-grade ureteric stent insertion was $83 \%$ and the failure rate was greatest (four of six) in cases of ureteric injury following gynecological procedures. There were no immediate complications. Sharma et al. ${ }^{[13]}$ concluded that: Ante-grade ureteric stenting can be used as a temporary or permanent solution to ureteric obstruction. The technique of insertion of ante-grade ureteric stent is safe, acceptable to patients and avoids the need for a general anesthetic. 
Jenkins and Marcus ${ }^{[14]}$ reported eleven kidneys in 10 patients in which nephrostomy insertion with subsequent immediate or delayed ante-grade ureteric stenting were undertaken. Ante-grade placement of the ureteric stent was achieved in 10 of the 11 kidneys (90\% success).

Lu et al. ${ }^{[15]}$ reviewed fifty consecutive per-cutaneous ureteric stent insertions in 40 patients. They reported that: Thirty seven of 50 cases were performed following failed retrograde-stenting. Ante-grade stenting failed in two out of 37 (5\%) of cases of malignant obstruction, and four out of 13 (31\%) cases of benign ureteral disease. Causes of failure and common technical problems included poor angulation of the per-cutaneous track, tortuous dilated ureters, tight obstruction, wedging of stent assembly components due to high resistance, and difficulty in positioning of the proximal pigtail. Helpful technical modifications included mid-pole rather than lower pole calyceal access, urinary decompression prior to stenting, and the routine use of a peel-away sheath (success rate 23/24 placement with sheath versus 21/26 without sheath). Stent patency rates were $95 \%$ at three months and $54 \%$ at six months following the stent insertions. Lu et al. ${ }^{[15]}$ concluded that with attention to technique and appropriate modification, success rate of per-cutaneous ureteric stenting remained high in their series.

Harding ${ }^{[16]}$ reported 34 successful per-cutaneous ante-grade ureteric stent placements from 37 attempts which were performed on 25 selected patients with a history of malignant diseases, in whom retrograde ureteric stenting was impossible or difficult. Harding ${ }^{[16]}$ stated this represented a $92 \%$ success rate. In their series the complication rate was $2 \%$ to $4 \%$.

Chitale et al. ${ }^{[17]}$ retrospectively, retrieved data from case notes and radiology data base of patients who had undergone one stage and two stage ante-grade ureteric stent insertion for the decompression of obstructed upper urinary tract. Chitale et al. ${ }^{[17]}$ reported that in relation to the results, a one-stage approach was found to be suitable in most cases and this approach had many advantages over the two-stage approach with comparable or better outcomes at lower costs.

The high success rate with regard to ante-grade ureteric stenting in our patients is encouraging which is consistent with the aforementioned reported series but the draw back in our study is the limited number of patients in that there were only 89 patients involved. Nevertheless, the high success rate would confirm that ante-grade ureteric stent insertion under local anesthesia is one of the useful options in the initial management of the obstructed upper urinary tract.

\section{Conclusions}

Our experience would confirm that insertion of ante-grade ureteric stent is a safe procedure with minimal complications.

The procedure is usually carried out without the need for general or spinal anaesthesia and it is well tolerated by adult patients of all age groups and sex.

The success rate of one-stage ante-grade ureteric stent insertions is high.

We would recommend that every radiology department should have a trained interventional radiologist who is capable of performing ante-grade ureteric stenting.

\section{Conflict of interests}

The authors declare that they have no conflict of interests.

\section{Acknowledgements}

The authors wanted to thank Dr. Emad Bakir, former consultant Radiologist at North Manchester General Hospital; Dr Eddie Tam, Consultant Radiologist at North Manchester General Hospital; and Dr. Z Sherazi, Consultant Radiologist at North Manchester General Hospital. 


\section{References}

[1] Venyo A, Bakir E. Antegrade Ureteric Stenting Prospective Experience in Managing 30 Patients; Indications; Complications And Outcome, Webmed Central UROLOGY. 2011; 2(1): WMC001442.

[2] Wah T M, Weston M J, Irving H C. Percutaneous nephrostomy insertion: outcome data from prospective multi-operator study at a UK training centre. Clin Radiol. 2004 Mar; 59(3): 255-261. PMid: 15037138. http://dx.doi.org/10.1016/j.crad.2003.10.021

[3] Goodwin W E, Casey W C, Woolf W. Per-cutaneous Trocar (needle) nephrostomy in hydronephrosis. JAMA. 1955 Mar 12; 157(11): 891-894. http://dx.doi.org/10.1001/jama.1955.02950280015005

[4] Farrell T A, Hicks M E. A review of radiologically guided percutaneous nephrostomies in 303 patients. J Vas Interv Radiol. 1997 Sep - Oct; 8(5): 769-774. http://dx.doi.org/10.1016/S1051-0443(97)70658-4

[5] Stables D P, Ginsburg N J, Johnson M L. Percutaneous nephrostomy: a series and review of the literature. A J R Am J Roentgenol. 1978 Jan; 130(1): 75-82. PMid: 413422. http://dx.doi.org/10.2214/ajr.130.1.75

[6] Paz A, Amiel G E, Pick N, Moskovitz B, Nativ O, Potasman I. Febrile complications following insertion of 100 double-J ureteral stents. J Endourol. 2005 Mar; 19(2): 147-150. PMid: 15798408. http://dx.doi.org/10.1089/end.2005.19.147

[7] Rao A A, Alleemudder A, Mukeril G, Mishra V, Motiwala H, Chari M, et al. Extra-anatomical complications of antegrade double-J insertion. Indian J Urol. 2011 Jan; 27(1): 19-24. http://dx.doi.org/10.4103/0970-1591.78408

[8] Liatsikos E N, Karnabatidis D, Katsanos K, Kallidonis P, Kadgadis G C, Christeas N, et al. Ureteral metal stents: 10-year experience with malignant ureteral obstruction treatment. J Urol. 2009 Dec; 182(6): 2613-2617. http://dx.doi.org/10.1016/j.juro.2009.08.040

[9] Kim B M, Park S I. Placement of double-J ureteric stent using the pull-through technique in patients with tight ureteric stents. Abdom Imaging. 2008 Mar-Apr; 33(2): 227-240. PMid: 17440767. http://dx.doi.org/10.1007/s00261-007-9233-1

[10] Watson G M, Patel U. Primary ante-grade ureteric stenting: prospective experience and cost-effectiveness analysis in 50 ureters. Clin Radiol. 2001 Jul; 56(7): 568-574. PMid: 11446755. http://dx.doi.org/10.1053/crad.2000.0708

[11] Hausegger K A, Portegaller H R. Percutaneous nephrostomy and ante-grade ureteral stenting: technique - indications complications. Eur Radiol. 2006 Sep; 16(9): 2016-2030. PMid: 16547709. http://dx.doi.org/10.1007/s00330-005-0136-7

[12] Borelli Palanca A, Ferrer Puchol M D, Villamón Fort R, Gil Romero J. Anterograde insertion of ureteral catheter. Actas Urol Esp. 2000 Mar; 24(3): 243-247. http://dx.doi.org/10.1016/S0210-4806(00)72439-9

[13] Sharma S D, Persad R A, Ha A, Appleton D S, Doyle P T, Bullock K N, et al. A review of antegrade stenting in the management of obstructed kidney Br J Urol. 1996 Oct; 78(4): 511-515. PMid: 8944505. http://dx.doi.org/10.1046/j.1464-410X.1996.01673.x

[14] Jenkins C N, Marcus A J. The value of antegrade stenting for lower ureteric obstruction. J R Soc Med. 1995 Aug; 86(8): 446-449.

[15] Lu D S, Papanicolaou N, Girad M, Lee M J, Yuder I C. Percutaneous internal ureteral stent placement: review of technical issues and solutions in 50 consecutive cases. Clin Radiol. 1994 Apr; 49(4): 256-261. http://dx.doi.org/10.1016/S0009-9260(05)81852-5

[16] Harding J R. Percutaneous antegrade ureteric stent insertion in malignant disease. J R Soc. Med. 1993 Sep; 86; 511-513. PMid: 8410885.

[17] Chitale S, Raja V, Hussain N, Saada J, Girling S, Irving S, et al. One-stage tubeless antegrade ureteric stenting: a safe and cost-effective option? Ann R Coll. Surg. Engl. 2009 Dec 7. Epub ahead of print URL. Available from: http://www.ncbi.nlm.nih.gov/pubmed/19995490?itool=EntrezSystem2.PEntraz.Pubm 